doi: $10.13108 / 2020-12-3-11$

\title{
SYNTHESIZABLE SEQUENCE AND PRINCIPLE SUBMODULES IN SCHWARTZ MODULE
}

\author{
N.F. ABUZYAROVA
}

\begin{abstract}
We consider a module of entire functions of exponential type and polynomial growth on the real axis, that is, the Schwarz module with a non-metrizable locally convex topology. In relation with the problem of spectral synthesis for the differentiation operator in the space $C^{\infty}(a ; b)$, we study principle submodules in this module. In particular, we find out what functions, apart of products of the polynomials on the generating function, are contained in a principle submodule. The main results of the work is as follows: despite the topology in the Schwarz module is non-metrizable, the principle submodule coincides with a sequential closure of the set of products of its generating function by polynomials. As a corollary of the main result we prove a weight criterion of a weak localizability of the principle submodule. Another corollary concerns a notion of "synthesizable sequence" introduced recently by A. Baranov and Yu. Belov. It follows from a criterion of the synthesizable sequence obtained by these authors that a synthesizable sequence is necessary a zero set of a weakly localizable principle submodule. In the work we give a positive answer to a natural question on the validity of the inverse statement. Namely, we prove that the weak set of a weakly localizable principle submodule is a synthesizable sequence.
\end{abstract}

Keywords: entire functions, Fourier-Laplace transform, Schwarz space, local description of submodules, spectral synthesis.

Mathematics Subject Classification: 30D15, 30H99, 42A38, 47E05

\section{INTRODUCTION}

Given a finite or an infinite interval $(a ; b) \subseteq \mathbb{R}$, we denote by $C^{\infty}(a ; b)$ the set of all infinitely differentiable functions equipped with a standard metrizable topology, while its strongly dual space consisting of all distributions compactly supported in in $(a ; b)$ is denoted by the symbol $\mathcal{E}^{\prime}(a ; b)$.

Let $W \subset \mathcal{E}(a ; b)$ be a closed subspace invariant with respect to the differentiation operator $D=\frac{\mathrm{d}}{\mathrm{d} t}$, or shortly, a $D$-invariant subspace. In work [1], the study of the problem on spectral synthesis was initiated and in particular, it was established that the spectrum $\sigma_{W}$ of the restriction of the differentiation operator $D: W \rightarrow W$ either coincides with entire complex plane or is discrete, that is, is an infinite of finite, probably, empty sequence of multiple points in $\mathbb{C}$ [1, Thm. 2.1].

For a non-empty relatively closed segment $I \subset(a ; b)$, the subspace $W_{I}$ is defined by the formula

$$
W_{I}=\{f \in \mathcal{E}(a ; b): f=0 \text { on } I\} .
$$

N.F. Abuzyarova, Synthesizable sequence and Principle submodules in Schwartz module.

(C) Abuzyarova N.F. 2020.

The research is made in the framework of the state task of the Ministry of Science and Higher Education of Russian Federation (code of scientific theme FZWU-2020-0027).

Submitted June 25, 2020. 
Each $D$-invariant subspace $W$ possesses a "residual" subspace $W_{\text {res }} \subset W$ being the maximal subspace of form (1.1) contained in $W$ [1, Thm. 4.1]. We denote a corresponding segment by $I_{W}$ and we call it residual segment of the subspace $W$, that is, $W_{\text {res }}=W_{I_{W}}$.

The existence of $D$-invariant subspaces of form (1.1) led the authors of work [1] to the following formulation of the problem on spectral synthesis: to find out under which conditions the $D$-invariant subspace $W$ with a discrete spectrum satisfies the representation

$$
W=\overline{W_{I_{W}}+\operatorname{span}(\operatorname{Exp} W)} ?
$$

Here $\operatorname{Exp} W$ is the set of all exponential monomials contained in $W$.

It turned out that in the case of a finite (in particular, empty) spectrum $\sigma_{W}$, the subspace $W$ is always of form (1.2), while if the spectrum $\sigma_{W}$ is discrete and infinite, then the answer depends on a relation between quantities $\left|I_{W}\right|$ and $2 \pi D_{B M}(\Lambda)$, where $\left|I_{W}\right|$ is the length of the residual segment, $D_{B M}(\Lambda)$ is the Beurling-Malliavin density of the set $\Lambda=\mathrm{i} \sigma_{W}$ :

1) if $\left|I_{W}\right|<2 \pi D_{B M}(\Lambda)$, then $W=\mathcal{E}(a ; b)$, see [2, Rem. 3], [3, Thm. 1.3]);

2) if $\left|I_{W}\right|=2 \pi D_{B M}(\Lambda)$, then there exist both $D$-invariant subspaces admitting spectral synthesis in a weak 1 sense $(1.2$, 4, [5] and subspaces not possessing this property [3], [6];

3 ) if $\left|I_{W}\right|>2 \pi D_{B M}(\Lambda)$, then $D$-invariant subspace with a discrete spectrum $\sigma_{W}=\mathrm{i} \Lambda$ and a residual segment $I_{W}$ admits a weak spectral synthesis (1.2) [2, Cor. 3], [3, Thm. 1.1].

The latter of the three above formulated results can be interpreted as follows: given a complex sequence $\Lambda$ and a relatively closed in $(a ; b)$ segment $I$ such that $|I|>2 \pi D_{B M}(\Lambda)$, there exists a unique $D$-invariant subspace $W \subset \mathcal{E}(a ; b)$ with a discrete spectrum $\sigma_{W}=-\mathrm{i} \Lambda$ and a residual segment $I_{W}=I$ and this subspace is of form $(1.2)$.

In view of this interpretation, the authors of work [7] called a sequence $\Lambda \subset \mathbb{C}$ with $D_{B M}(\Lambda)<$ $+\infty$ syntheziable if a $D$-invariant subspace with a spectrum - $(\mathrm{i} \Lambda)$ and a residual segment $\left[-\pi D_{B M}(\Lambda) ; \pi D_{B M}(\Lambda)\right]$ is unique; in this case it is of form (1.2). In that work a complete description of synthesizable sequences was provided. In particular, it was shown that the if the system of exponential monomials $\operatorname{Exp}_{\Lambda}$ constructed by the sequence $-(\mathrm{i} \Lambda)$ is complete or it has a finite defect in the space $L^{2}\left(-\pi D_{B M}(\Lambda) ; \pi D_{B M}(\Lambda)\right)$, then $\Lambda$ is a synthesizable sequence [7, Prop. 3.2].

If the system $\operatorname{Exp}_{\Lambda}$ has an infinite defect in $L^{2}\left(-\pi D_{B M}(\Lambda) ; \pi D_{B M}(\Lambda)\right)$, then the syntesizability of $\Lambda$ is determined by the conditions of the following criterion [7, Thm. 1.3]:

Theorem A. A sequence $\Lambda \subset \mathbb{C}$ is synthesizable if and only if it is a zero set of some function $\varphi \in \mathcal{P}_{0}(\mathbb{R})$ and

$$
\operatorname{dim}\left(\mathcal{H}(\varphi) \ominus H_{\text {pol }}\right) \leqslant 1 .
$$

In the formulation of the above theorem we have employed the following notations: $\mathcal{P}_{0}(\mathbb{R})$ is the set of all entire functions $\varphi$ of exponential type, the indicators of which satisfy the estimates

$$
h_{\varphi}(\arg z) \leqslant C_{\varphi}|\operatorname{Im} z|, \quad z \in \mathbb{C},
$$

and on the real axis the identity holds:

$$
|\varphi(x)|=o\left(|x|^{-n}\right), \quad|x| \rightarrow \infty, \quad n=1,2, \ldots ;
$$

$\mathcal{H}(\varphi)$ is a Hilbert space consisting of all entire functions $\omega$ of minimal type at order 1 such that

$$
\int_{-\infty}^{\infty}|\omega(x) \varphi(x)|^{2} \mathrm{~d} x<+\infty
$$

equipped with the scalar product

$$
\left(\omega_{1}, \omega_{2}\right)=\int_{\mathbb{R}} \omega_{1}(x) \overline{\omega_{2}(x)}|\varphi(x)|^{2} \mathrm{~d} x, \quad \omega_{1}, \omega_{2} \in \mathcal{H}(\varphi),
$$

\footnotetext{
1"weak" with respect to the classical spectral synthesis when $W=\overline{\operatorname{span}(\operatorname{Exp} W)}$
} 
$H_{\text {pol }}$ is the closure of the set of polynomials in $\mathcal{H}(\varphi)$.

It is clear that the synthesizability of a sequence $\Lambda$ is a sufficient condition for admitting a weak spectral synthesis by a $D$-invariant subspace with a discrete spectrum $-(\mathrm{i} \Lambda)$ and a residual segment of length $2 \pi D_{B M}(\Lambda)$. This gives rise to a question: when the syntesizability of a sequence $\Lambda$ is also a necessary condition for admittance of a weak spectral synthesis by a $D$-invariant subspace with the spectrum - $(\mathrm{i} \Lambda)$ and a residual segment equalling to $\left[-\pi D_{B M}(\Lambda) ; \pi D_{B M}(\Lambda)\right]$ (or to any other fixed segment of the length $2 \pi D_{B M}(\Lambda)$ )?

One of the aims of the present work is to answer this question.

Earlier for studying $D$-invariant subspaces we employed effectively the scheme of dual spaces reducing the problem on subspaces to equivalent problems on closed submodules in a special module of entire functions $\mathcal{P}(a ; b)$, see [2], [4], [8]. We are going to employ this scheme in the present work and this is why we describe briefly the duality between $D$-invaiant subspaces and submodules.

For each element $S \in \mathcal{E}^{\prime}(a ; b)$ we introduce its Fourier-Laplace transform

$$
\mathcal{F}(S)(z)=S\left(e^{-i t z}\right), \quad z \in \mathbb{C},
$$

which is an entire function of a completely regular growth at order 1 . We denote it by $\varphi$. The indicator diagram of the function $\varphi$ is the segment of the imaginary axis

$$
\mathrm{i}\left[c_{\varphi} ; d_{\varphi}\right] \subset \mathrm{i}(a ; b),
$$

where $c_{\varphi}=-h_{\varphi}(-\pi / 2), d_{\varphi}=h_{\varphi}(\pi / 2)$, and $h_{\varphi}$ is the indicator of the function $\varphi$.

We let $\mathcal{P}(a ; b)=\mathcal{F}\left(\mathcal{E}^{\prime}(a ; b)\right)$. It is well known that $\mathcal{P}(a ; b)=\bigcup P_{k}$, where $\left\{P_{k}\right\}$ is an increasing sequence of Banach spaces, each being the set of all entire functions $\varphi$ with a finite norm

$$
\|\varphi\|_{k}=\sup _{z \in \mathbb{C}} \frac{|\varphi(z)|}{(1+|z|)^{k} \exp \left(b_{k} y^{+}-a_{k} y^{-}\right)}, \quad y^{ \pm}=\max \{0, \pm y\}, \quad z=x+\mathrm{i} y,
$$

$\left[a_{1} ; b_{1}\right] \Subset\left[a_{2} ; b_{2}\right] \Subset \ldots$ is a sequence of segments exhausting the interval $(a ; b)$. Equipping the set $\mathcal{P}(a ; b)$ by a locally convex topology of the inductive limit of the sequence $\left\{P_{k}\right\}$, we obtain a space of type $\left(L N^{*}\right)$, see [9], isomorphic to $\mathcal{E}^{\prime}(a ; b)$ [10, Thm. 7.3.1]. We note that according to the same theorem, $\mathcal{P}_{0}(\mathbb{R})=\mathcal{F}\left(C_{0}^{\infty}(\mathbb{R})\right)$.

In the space $\mathcal{P}(a ; b)$, the operation of multiplication by an independent variable $z$ is continuous and this is why $\mathcal{P}(a ; b)$ is a topological module over the ring of polynomials $\mathbb{C}[z]$ called Schwartz module.

A closed submodule $J \subset \mathcal{P}(a ; b)$ is a closed subspace satisfying also the condition $z J \subset J$. In what follows, for the sake of brevity, we shall say "submodule" meaning a closed submodule.

We recall a series of notions characterising submodules, see [11], [12]. An indicator segment of a submodule $J$ is the segment $\left[c_{J} ; d_{J}\right] \subset \overline{\mathbb{R}}$, where $c_{J}=\inf _{\varphi \in J} c_{\varphi}, d_{J}=\sup _{\varphi \in J} d_{\varphi}$.

A divisor of a submodule $J \subset \mathcal{P}(a ; b)$ is a function $n_{J}(\lambda)=\min _{\varphi \in J} n_{\varphi}(\lambda), \lambda \in \mathbb{C}$, where $n_{\varphi}(\lambda)$ is a divisor of the function $\varphi \in J$ :

$$
n_{\varphi}(\lambda)= \begin{cases}0 & \text { if } \quad \varphi(\lambda) \neq 0, \\ m & \text { if } \quad \lambda \text { is a zero of } \varphi \text { of multiplicity } m\end{cases}
$$

and

$$
\Lambda_{\varphi}=\left\{\lambda \in \mathbb{C}: n_{\varphi}(\lambda)>0\right\}, \quad \Lambda_{J}=\left\{\lambda \in \mathbb{C}: n_{J}(\lambda)>0\right\}
$$

are zero sets of the function $\varphi$ and submodule $J$, respectively, and each point $\lambda$ is repeated according its multiplicity.

The submodules of the module $\mathcal{P}(a ; b)$ are dual to $D$-invariant subspaces of the space $\mathcal{E}(a ; b)$. Namely, there exists an one-to-one correspondence between the set of closed submodules $\{J\}$ of the module $\mathcal{P}(a ; b)$ and the of $D$-invariant subspaces $\{W\}$ of the space $\mathcal{E}(a ; b)$. This one-to-one 
correspondence is defined by the following rule: $J \longleftrightarrow W$ if and only if $J=\mathcal{F}\left(W^{0}\right)$, where a closed subspace $W^{0} \subset \mathcal{E}^{\prime}(a ; b)$ consists of all distributions $S \in \mathcal{E}^{\prime}(a ; b)$ annihilating $W$; here

$$
\operatorname{Exp} W=\left\{t^{j} e^{-\mathrm{i} \lambda_{k} t}, \quad j=0, \ldots m_{k}-1, \quad n_{J}\left(\lambda_{k}\right)=m_{k}>0\right\},
$$

and the points $c_{J}$ and $d_{J}$ serve as boundaries for the residual segment $I_{W}$, see [2], [12]. The above formulated fact is called the duality principle.

A submodule $J \subset \mathcal{P}(a ; b)$ is weakly localizable if for each function $\varphi \in \mathcal{P}(a ; b)$ the conditions

1) $n_{\varphi}(z) \geqslant n_{J}(z)$ for all $z \in \mathbb{C}$,

2) the indicator diagram of the function $\varphi$ is contained in the set $\mathrm{i}\left[c_{J} ; d_{J}\right]$; imply that $\varphi \in J$.

A submodule $J$ is called stable if for each $\lambda \in \mathbb{C}$ an implication holds:

$$
\varphi \in J, \quad n_{\varphi}(\lambda)>n_{J}(\lambda) \Longrightarrow \frac{\varphi}{z-\lambda} \in J .
$$

A $D$-invariant subspace $W$ admits a weak spectral synthesis if and only if its annihilating submodule $\mathcal{J}=\mathcal{F}\left(W^{0}\right)$ is weakly localizable, see [2], 4].

A $D$-invariant subspace $W$ has a discrete spectrum if and only if its annihilating submodule $\mathcal{J}=\mathcal{F}\left(W^{0}\right)$ is stable [1, Prop. 3.1], [12, Prop. 2].

A principle submodule $J_{\varphi}$ generated by a function $\varphi \in \mathcal{P}(a ; b)$ is defined as a closure of the set

$$
\operatorname{Pol}_{\varphi}=\{p \varphi: \quad p \in \mathbb{C}[z]\}
$$

in $\mathcal{P}(a ; b)$. A principle submodule is always stable [12].

Let, as above, $\Lambda$ be a complex sequence with a finite Beurling-Malliavin density; $W \subset \mathcal{E}(\mathbb{R})$ be a $D$-invariant subspace with the spectrum $\sigma_{W}=-\mathrm{i} \Lambda$ and the residual segment $I_{W}=$ $\left[-\pi D_{B M}(\Lambda) ; \pi D_{B M}(\Lambda)\right]$. We observe that if the residual segment $I_{W}$ is given, the corresponding subspace $W$ can be considered in each space $\mathcal{E}(a ; b)$ such that $I_{W} \subset(a ; b)$.

We assume first that the sysmte $\operatorname{Exp}_{\Lambda}$ is either complete or have a finite defect in the space $L^{2}\left(-\pi D_{B M}(\Lambda) ; \pi D_{B M}(\Lambda)\right)$. It is easy to make sure that this is equivalent to the existence of the function $\varphi \in \mathcal{P}(\mathbb{R}) \backslash \mathcal{P}_{0}(\mathbb{R})$, with the zero set $\Lambda_{\varphi}=\Lambda$ and the indicator diagram $\left[-\mathrm{i} \pi D_{B M}(\Lambda) ; \mathrm{i} \pi D_{B M}(\Lambda)\right]$. By Theorem 2 in work [8], this implies that the annihilator submodule of the subspace $W$ is the principle submodule $\mathcal{J}_{\varphi}$ with generator $\varphi$. Moreover, in this case,

$$
\mathcal{J}(\varphi)=\mathcal{J}_{\varphi}=\{p \varphi: \quad p \in \mathbb{C}[z]\}
$$

where the symbol $\mathcal{J}(\varphi)$ denotes a weakly localizable sudmodule with the zero set $\Lambda$ and the indicator segment $\left[-\mathrm{i} \pi D_{B M}(\Lambda) ; \mathrm{i} \pi D_{B M}(\Lambda)\right]$.

By the duality between $D$-invariant subspaces and submodules and by the said above Theorem $\mathrm{A}$ we conclude that if the system $\operatorname{Exp}_{\Lambda}$ is complete or has a finite defect in the space

$$
L^{2}\left(-\pi D_{B M}(\Lambda) ; \pi D_{B M}(\Lambda)\right),
$$

then $W$ admits a weak spectral synthesis and $\Lambda$ is a synthesizable sequence.

Now we consider the case when the exponential system $\operatorname{Exp}_{\Lambda}$ has an infinite defect in $L^{2}\left(-\pi D_{B M}(\Lambda) ; \pi D_{B M}(\Lambda)\right)$. In this case it follows from Theorem A and the duality that for the synthesizability of $\Lambda$, it is necessary that the space $W$ is of the form

$$
W=W_{S}=\left\{f \in \mathcal{E}(\mathbb{R}): \quad S\left(f^{(k)}\right)=0 \quad \text { for all } k=0,1, \ldots\right\},
$$

where $S \in \mathcal{E}^{\prime}(\mathbb{R})$, and $\varphi=\mathcal{F}(S) \in \mathcal{P}_{0}(\mathbb{R}), \Lambda_{\varphi}=\Lambda$, while the indicator diagram $\varphi$ is $\left[-\mathrm{i} \pi D_{B M}(\Lambda) ; \mathrm{i} \pi D_{B M}(\Lambda)\right]$. Then $\mathcal{F}\left(W_{S}^{0}\right)=\mathcal{J}_{\varphi}$ and the admittance of the weak spectral synthesis for $W_{S}$ is equivalent to the weak localizability of $\mathcal{J}_{\varphi}: \mathcal{J}_{\varphi}=\mathcal{J}(\varphi)$. In other words, it 
is equivalent to the fact that $\mathcal{J}(\varphi)$ is the closure of the set $\mathrm{Pol}_{\varphi}$ in the topology of the space $\mathcal{P}(\mathbb{R})$.

On the other hand, in the considered case, if $\Lambda$ is a synthesizable sequence, then the submodule $\mathcal{J}(\varphi)$ coincides with a sequential closure of the set $\operatorname{Pol}_{\varphi}$, that is, with the set of all limits of countable sequences in $\mathrm{Pol}_{\varphi}$ converging in the topology of the space $\mathcal{P}(\mathbb{R})$; this set is indicated by the symbol $\mathcal{J}_{\varphi, s e q}$. This is implied by Theorem A in view of the remark after Lemma 1 , see the next section.

Thus, for the equivalence of the synthesizability of the sequence $\Lambda$ and the admittance of a weak spectral synthesis by the corresponding $D$-invariant subspace $W$ with the spectrum $\sigma_{W}=-\mathrm{i} \Lambda$ and the residual segment $I_{W}=\left[-\pi D_{B M}(\Lambda) ; \pi D_{B M}(\Lambda)\right]$, it is necessary that $W=W_{S}$ and $\mathcal{J}_{\varphi}=\mathcal{J}_{\varphi, \text { seq }}$, where $\varphi=\mathcal{F}(S)$, and $\Lambda_{\varphi}=\Lambda$, and the indicator diagram of $\varphi$ is the segment $\left[-\mathrm{i} \pi D_{B M}(\Lambda) ; \mathrm{i} \pi D_{B M}(\Lambda)\right]$.

The space $\mathcal{P}(a ; b)$ is non-metrizable [9, Cor. 2 of Thm. 1]. This is why, generally speaking, the closure of an arbitrary set $A \subset \mathcal{P}(a ; b)$ can not be obtained just by adding the limits of converging countable sequences $\left\{\varphi_{n}\right\} \subset A$. Therefore, to answer the question on equivalence of synthesizability of the sequence $\Lambda$ and the weak spectral synthesis for the corresponding subspace of form (1.6) with the spectrum $\sigma_{W}=-\mathrm{i} \Lambda$, we first need to study whether the identity

$$
J_{\varphi, s e q}=J_{\varphi}
$$

is possible.

Theorem 1. Identity (1.7) holds for all $\varphi \in \mathcal{P}(a ; b)$.

By means of this theorem we prove the equivalence of the synthesizability of the sequence $\Lambda$ and the admittance of the weak spectral synthesis by a space of form $(1.6)$ with the spectrum $-(\mathrm{i} \Lambda)$, see Corollary 2 . Another important application of Theorem 11 is a convenient weight criterion of the weak localizability of the principle submodule in the module $\mathcal{P}(a ; b)$, see Theorem 2,

The main results of the present work were announced in [13].

2. Sequential Description of PRinciple submodules in the Schwartz module

2.1. Preliminaries. Let $[c ; d] \subset(a ; b), P W(c ; d)=\mathcal{F}\left(L^{2}(c ; d)\right)$ be the Paley-Wiener space, $P_{0}[c ; d]$ be the space of all entire functions $\psi$ with a finite norm

$$
\|\psi\|_{0}=\sup _{z \in \mathbb{C}} \frac{|\psi(z)|}{\exp \left(d y^{+}-c y^{-}\right)}, \quad y^{ \pm}=\max \{0, \pm y\}, \quad z=x+\mathrm{i} y .
$$

Lemma 1. If $\psi \in P W(c ; d)$, then $\psi \in P_{0}[c ; d]$, and

$$
\|\psi\|_{0} \leqslant C_{0}\|\psi\|_{P W(c ; d)},
$$

where a positive constant $C_{0}$ is independent of $c$ and $d$.

Proof. Without loss of generality we can assume that $c=-d$; then

$$
\begin{aligned}
& \psi(z)=\int_{-d}^{d} e^{-\mathrm{i} z t} f(t) \mathrm{d} t, \quad f \in L^{2}(-d ; d), \\
& \|\psi\|_{0}=\sup _{z \in \mathbb{C}} \frac{|\psi(z)|}{\exp (d|y|)}, \quad z=x+\mathrm{i} y .
\end{aligned}
$$

According Plancherel theorem, for a fixed $y \in \mathbb{R}$ we have

$$
\|\psi(x+\mathrm{i} y)\|_{L^{2}(\mathbb{R})}^{2}=2 \pi\left\|e^{y t} f(t)\right\|_{L^{2}(-d ; d)}^{2} .
$$


Employing this fact and a subharmonicity of the function $|\psi|^{2}$, for all $x \in \mathbb{R}$ we obtain the estimates

$$
|\psi(x)|^{2} \leqslant \frac{1}{\pi} \int_{|w-x| \leqslant 1}|\psi(w)|^{2}|\mathrm{~d} w| \leqslant \frac{1}{\pi} \int_{-1}^{1}\left(\int_{-\infty}^{+\infty}|\psi(s+\mathrm{i} \tau)|^{2} \mathrm{~d} s\right) \mathrm{d} \tau \leqslant C_{1} e^{2 d}\|\psi\|_{P W(-d ; d)}^{2},
$$

where $C_{1}$ is an absolute constant. Inequality (2.2) follows from these estimates and PhragménLindelöf principle.

Remark 1. Theorem $A$ and the proven Lemma yield easily that if the zero set $\Lambda_{\varphi}$ of the function $\varphi \in \mathcal{P}(a ; b) \bigcap \mathcal{P}_{0}(\mathbb{R})$ is a synthesizable sequence, then

$$
\mathcal{J}(\varphi)=\mathcal{J}_{\varphi}=\mathcal{J}_{\varphi, s e q}
$$

Indeed, if $\Phi \in \mathcal{J}(\varphi)$, then $\Phi=\omega \varphi$, where $\omega$ is an entire function of the minimal type and there exists a polynomial $q_{\Phi}$ such that $\frac{\omega}{q_{\Phi}} \in \mathcal{H}(\varphi)$. Then, by Theorem A, either $\frac{\omega}{q_{\Phi}} \in H_{\text {pol }}$ or for an arbitrary fixed point $\lambda_{0} \in \Lambda_{\omega} \backslash \Lambda_{\varphi}$ there exist numbers $\alpha_{1}, \alpha_{2} \in \mathbb{C}$ such that

$$
\left(\alpha_{2}-\frac{\alpha_{1}}{z-\lambda_{0}}\right) \cdot \frac{\omega}{q_{\Phi}} \in H_{p o l} .
$$

In both case, in view of the intrinsic description of the space $\mathcal{P}(a ; b)$ and a sequential convergence in it, [9, Cor. 1 from Thm. 2], by the proven lemma we conclude that $\Phi \in \mathcal{J}_{\varphi, s e q}$.

Let $\varphi \in \mathcal{P}_{0}(\mathbb{R}), c_{\varphi}=h_{\varphi}(-\pi / 2), d_{\varphi}=h_{\varphi}(\pi / 2)$, where $h_{\varphi}$ is the indicator of the function $\varphi, P W=P W\left(c_{\varphi} ; d_{\varphi}\right)$. We consider the following closed subspaces in $P W$ : the subspace $P W(\varphi)=J(\varphi) \bigcap P W$ and the subspace $P W_{\text {pol }}$ defined as the closure of the set $\mathrm{Pol}_{\varphi}$ in $P W$.

A one-to-one correspondence

$$
\omega \mapsto \omega \varphi, \quad \omega \in \mathcal{H}(\varphi),
$$

makes an isometry of Hilbert spaces $\mathcal{H}(\varphi)$ and $P W(\varphi)$. The subspace $H_{\text {pol }}$ defined as the closure of the set of polynomials in $\mathcal{H}(\varphi)$ is the pre-image of the subspace $P W_{\text {pol }}$ under this isometry.

We shall need some definitions and facts from the general theory of de Branges spaces [14], and also from work [7, in which this theory was successfully employed for studying $D$-invariant subspaces in the Schwartz space (in particular, for the proof of Theorem A).

Originally, de Branges space is defined as associated with an entire function $E$ from the Hermite-Biehler class and is the set of all entire functions $F$, such that

$$
\int_{-\infty}^{+\infty}\left|\frac{F(t)}{E(t)}\right|^{2} \mathrm{~d} t<+\infty
$$

and obeying some further restrictions, see [14, Sects. 19-21], [7, Sect. 2]).

In this work, we restrict ourselves by an exact formulation of an equivalent definition of de Branges space; this definition is an axiomatic description, see [14, Thm. 23]): a non-trivial Hilbert space of entire functions $\mathcal{H}$ is a de Branges space if and only if the following axioms are satisfied:

$(H 1)$ if $F \in \mathcal{H}, \lambda \in \mathbb{C} \backslash \mathbb{R}$ is a zero of the function $F$, then $F_{1}=F(z) \frac{z-\bar{\lambda}}{z-\lambda} \in \mathcal{H}$ and the norm of the funcionts $F$ and $F_{1}$ are equal;

(H2) for each $\lambda \in \mathbb{C} \backslash \mathbb{R}$, a corresponding linear $\delta_{\lambda}$-functional acting by the rule $\delta_{\lambda}(F)=F(\lambda)$, $F \in \mathcal{H}$, is continuous in $\mathcal{H}$;

(H3) for each function $F \in \mathcal{H}$, the function $F^{*}(z)=\overline{F(\bar{z})}$ belongs to $\mathcal{H}$ and has the same norm as $F$.

By means of this axiomatic description, it was established in [15], [7, Sect. 2, Thm. 2.7] that $\mathcal{H}(\varphi)$ is a de Branges space. It is also easy to check that axioms (H1)-(H3) holds also for 
the subspace $H_{p o l}$ regarded as a Hilbert space with scalar product (1.3), that is, $H_{\text {pol }}$ is a de Branges space.

We also formulate two results on de Branges space, see [14, Sect. 35], [7, Thm. 2.1] and [14, Sect. 29], respectively.

Theorem B. Let $H_{1}$ and $H_{2}$ be closed subspaces of the same de Branges space $\mathcal{H}$ being also de Branges spaces with the scalar product induced from $\mathcal{H}$. Then one of the following inclusions holds: $H_{1} \subset H_{2}$ or $H_{2} \subset H_{1}$.

Theorem C. Let $\mathcal{H}$ be a de Branges space, $H_{k}$ be the closure of a linear set $\left\{f \in \mathcal{H}: z^{j} f \in\right.$ $\mathcal{H}, j=1, \ldots, k\}, k \in \mathbb{N}$, in $\mathcal{H}$. Then $\operatorname{dim}\left(\mathcal{H} \ominus H_{k}\right)<+\infty$.

Employing Theorems B and C, it is easy to prove the following lemma.

Lemma 2. Assume that in the space $H_{\text {pol }}$ there exists a function $\omega_{0}$ with the following property:

for some $k_{0} \in \mathbb{N}$. Then

$$
z^{k_{0}-1} \omega_{0} \in \mathcal{H}(\varphi), \quad z^{k_{0}} \omega_{0} \notin \mathcal{H}(\varphi)
$$

$$
\operatorname{dim}\left(\mathcal{H}(\varphi) \ominus H_{\text {pol }}\right) \leqslant 1
$$

Proof. For each $k \in \mathbb{N}$, by the symbol $\mathcal{H}_{k}$ we denote the closure of the set

$$
\left\{\omega \in \mathcal{H}(\varphi): z^{k} \omega \in \mathcal{H}(\varphi)\right\}
$$

in $\mathcal{H}(\varphi)$.

Since $\mathcal{H}(\varphi)$ is the pre-image of the set $P W \cap \mathcal{J}(\varphi)$ under the isometry $(2.3)$, and $\mathcal{J}(\varphi)$ is a stable submodule, then $\mathcal{H}_{k}$ coincides with the subspace $H_{k}$ from Theorem $\mathrm{C}$. It is also clear that $\mathcal{H}_{0}=\mathcal{H}(\varphi), \mathcal{H}_{k} \subset \mathcal{H}_{k-1}, k=1,2, \ldots$

Each $\mathcal{H}_{k}$ with the scalar product induced by that in $\mathcal{H}(\varphi)$ is a de Branges space, as well as the subspace $H_{p o l}$. This is why by Theorem B, either $\mathcal{H}_{k_{0}} \subset H_{p o l}$ or $H_{p o l} \subset \mathcal{H}_{k_{0}}$. But the presence of the function $\omega_{0}$ in $H_{\text {pol }}$ excludes the possibility $H_{p o l} \subset \mathcal{H}_{k_{0}}$; therefore,

$$
\mathcal{H}_{k_{0}} \subset H_{\text {pol }} \text {. }
$$

In view of Theorem $\mathrm{C}$ we have

$$
\operatorname{dim}\left(\mathcal{H}(\varphi) \ominus H_{\text {pol }}\right) \leqslant \operatorname{dim}\left(\mathcal{H}(\varphi) \ominus \mathcal{H}_{k_{0}}\right)<+\infty .
$$

On the other hand, it is known that the codimension of $H_{\text {pol }}$ in $\mathcal{H}(\varphi)$ can take only three possible values: $0,1,+\infty$ [7, Thms. 2.1, 2.2, 2.9]. This implies the desired statement.

2.2. Proof of Theorem $\mathbf{1}$. As it has been already mentioned in the Introduction, by Theorem 2 in [8], the relation $\varphi \in \mathcal{P}(a ; b) \backslash \mathcal{P}_{0}(\mathbb{R})$ is equivalent to the validity of 1.5 and hence, in this case the statement of the theorem is trivial.

Let $\varphi \in \mathcal{P}(a ; b) \bigcap \mathcal{P}_{0}(\mathbb{R})$. Then, as it has been said in the end of the proof of Lemma 2 , the quantity $\operatorname{dim}\left(\mathcal{H}(\varphi) \ominus H_{\text {pol }}\right)$ can take only one of three possible values: $0,1,+\infty$.

If $\operatorname{dim}\left(\mathcal{H}(\varphi) \ominus H_{\text {pol }}\right)=0$, then

$$
J_{\varphi, s e q}=J_{\varphi}=J(\varphi)
$$

In the case $\operatorname{dim}\left(\mathcal{H}(\varphi) \ominus H_{\text {pol }}\right)=1$, identities $(2.4)$ can be proved on the base of Lemma 1 by arguing in the same way as in the remark after this lemma.

We consider the last option:

$$
\operatorname{dim}\left(\mathcal{H}(\varphi) \ominus H_{\text {pol }}\right)=+\infty
$$

We denote by $H_{\varphi}$ the pre-image of the closed subspace $P W_{\varphi}=P W \cap \mathcal{J}_{\varphi}$ of the space $P W$ under isometry (2.3) and we let $H_{1}=H_{\varphi} \ominus H_{\text {pol }}$. 
To complete the proof of the theorem, it is sufficient to make sure that

$$
H_{1}=\{\overline{0}\} .
$$

First of all we observe that the subspace $H_{1}$ can not contain a non-zero function $\omega$ satisfying $\Phi=\omega \varphi \in \mathcal{P}_{0}(\mathbb{R})$. Indeed, otherwise

$$
\Phi=\mathcal{F}(s), \quad s \in C_{0}^{\infty}(\mathbb{R}) \bigcap \mathcal{E}^{\prime}(a ; b) .
$$

And if $S_{\varphi}$ is a regular functional belonging to in $C_{0}^{\infty}(a ; b)$ obeying the identity $\varphi=\mathcal{F}\left(S_{\varphi}\right)$, then

$$
\int_{a}^{b} S_{\varphi}^{(k)}(t) \overline{s(t)} \mathrm{d} t=0, \quad k=0,1,2 \ldots
$$

Therefore, $\bar{s} \in W_{S_{\varphi}}$.

On the other hand, $\Phi \in J_{\varphi}$, since $\omega \in H_{1} \subset H_{\varphi}$. This is why $s \in W_{S_{\varphi}}^{0}$ and

$$
0=s(\bar{s})=\int_{a}^{b} s(t) \overline{s(t)} \mathrm{d} t
$$

that is, $s=0$. Thus, if $\omega \in H_{1} \backslash\{\overline{0}\}$, then there exists a number $n_{\omega} \in \mathbb{N}$ such that

$$
z^{j} \omega \in \mathcal{H}(\varphi), \quad j=0, \ldots, n_{\omega}-1, \quad z^{n_{\omega}} \omega \notin \mathcal{H}(\varphi) .
$$

Suppose we shall succeed to establish the following fact.

(F): in the subspace $H_{\text {pol }}$, there exists a function with property (2.6).

Applying then Lemma 2, we conclude that $\operatorname{dim}\left(\mathcal{H}(\varphi) \ominus H_{\text {pol }}\right)<+\infty$, and this contradicts relation (2.5). Thus, we have established that in case $(2.5)$ we have

$$
H_{1}=\{\overline{0}\}, \quad J_{\varphi, s e q}=J_{\varphi} \neq J(\varphi),
$$

that is, the principle submodule $J_{\varphi}$ is sequentially generated but is not weakly localizable.

It remains to justify statement $(\mathbf{F})$.

Let $\left\{\mu_{j}\right\}$ be a "sparse" sequence of zeroes a fixed non-zero function $\omega \in H_{1}$, say, such that $\mu_{1}>1, \mu_{j}>8 \mu_{j-1}, j=2,3, \ldots$ We let

$$
q_{m}(z)=\prod_{j=1}^{m}\left(1-\frac{z}{\mu_{j}}\right), \quad \tilde{\omega}_{m}=\frac{\omega}{q_{m}} .
$$

It is clear that $\tilde{\omega}_{m}$ satisfies condition (2.6) and by the stability of the submodule $J_{\varphi}$ we have $\tilde{\omega}_{m} \in H_{\varphi}$.

Let $\operatorname{Pr}_{p o l}: H_{\varphi} \rightarrow H_{p o l}$ and $\operatorname{Pr}_{1}: H_{\varphi} \rightarrow H_{1}$ be the projectors on the corresponding subspaces. If $\operatorname{Pr}_{1}\left(\tilde{\omega}_{m}\right)=0$ for some index $m$, then statement $(\mathbf{F})$ holds. Otherwise $\operatorname{Pr}_{1}\left(\tilde{\omega}_{m}\right) \neq 0$ for all $m=1,2, \ldots$ Employing standard ways for estimating entire functions and for description of bounded sets in locally-convex spaces of type $\left(L N^{*}\right)$ [9, Thm. 2], the space $\mathcal{P}(a ; b)$ being one of those, it is easy to confirm that the sequence $\left\{\tilde{\omega}_{m} \varphi\right\}$ is bounded in the sense of some norm $\|\cdot\|_{k_{0}}$, see (1.4). Hence, there exists a subsequence converging in $\mathcal{P}(a ; b)$, more precisely,

$$
\left\|\tilde{\omega}_{m_{j}} \varphi-\tilde{\omega}_{0} \varphi\right\|_{k_{0}+1} \rightarrow 0
$$

where

$$
\tilde{\omega}_{0}(z)=\frac{\omega(z)}{\prod_{i=1}^{\infty}\left(1-\frac{z}{\mu_{i}}\right)} .
$$

Let $q$ be some polynomial of degree $\left(k_{0}+2\right)$ with roots in the set $\Lambda_{\omega} \backslash\left\{\mu_{j}\right\}$. As in the case of $\tilde{\omega}_{m}$, if $\operatorname{Pr}_{1}\left(\tilde{\omega}_{m} q^{-1}\right)=0$ for some index $m$, then $\tilde{\omega}_{m} q^{-1} \in H_{\text {pol }}$ satisfies $(2.6)$ and statement (F) holds. Otherwise we employ the convergence of the sequence $\left\{\tilde{\omega}_{m_{j}} q^{-1}\right\}$ converges to the function $\omega_{0}=\tilde{\omega}_{0} q^{-1}$ in the space $\mathcal{H}(\varphi)$ and $\omega_{0} \varphi \in \mathcal{P}_{0}(\mathbb{R})$. By the above remark that each 
function in $H_{1}$ satisfies $(2.6)$, we have $\operatorname{Pr}_{\text {pol }}\left(\omega_{0}\right) \neq 0$. If $\operatorname{Pr}_{1}\left(\omega_{0}\right) \neq 0$, then the function $\operatorname{Pr}_{\text {pol }}\left(\omega_{0}\right)$ is the sought one and ( $\mathbf{F})$ holds.

It remains to treat the case $\omega_{0} \in H_{\text {pol }}$. We observe that multiplying the function $\omega_{0}$ by arbitrary rational function $Q$ such that $Q \omega_{0}$ is entire produces a function belonging to $H_{\varphi}$ and not satisfying condition (2.6). This is why, if for some rational function $Q_{0}$ the inequality $\operatorname{Pr}_{1}\left(Q_{0} \omega_{0}\right) \neq 0$ holds, then the function $\operatorname{Pr}_{p o l}\left(Q_{0} \omega_{0}\right)$ satisfies $(\mathbf{F})$.

Finally, let $Q \omega_{0} \in H_{\text {pol }}$ for each rational function $Q$ such that $Q \omega_{0}$ is entire. For the principle submodule generated by the function

$$
\Phi=\omega q^{-1} \varphi
$$

relations 1.5 hold since the function $\omega q^{-1}$ satisfies 2.6 . In view of the restrictions determining the choice of the points $\left\{\mu_{j}\right\}$, now we are under the same conditions as before Theorem 2 in work [8, Sect. 2]. Employing then Lemmata 1-3 of this work, we find a sequence of polynomials $\left\{p_{j}\right\}$ such that

$$
\lim _{j \rightarrow \infty} p_{j} \omega_{0} \varphi=\Phi
$$

in the space $\mathcal{P}(a ; b)$. In view of the description of the sequential convergence in $\mathcal{P}(a ; b)$, see $[9$, Cor. 1 from Thm. 2], we conclude that there exists a polynomial $p$ possessing the following property: the sequence $\left\{p_{j} \omega_{0} p^{-1}\right\}$ converges to an entire function $\nu=\omega q^{-1} p^{-1}$ in the norm of the space $\mathcal{H}(\varphi)$ and the function $\nu$ satisfies $(2.6)$. Since $\operatorname{Pr}_{1}\left(p_{j} \omega_{0} p^{-1}\right)=0$ for all values of the index $j$, then $\nu \in H_{\text {pol }}$ and this completes the proof.

\section{APPLICATION OF MAIN RESUlT}

Let $\Lambda \subset \mathbb{C}, 2 \pi D_{B M}(\Lambda)<b-a$. By Theorem 1 and Theorem A we obtain the following statement.

Corollary 1. A stable submodule $J \subset \mathcal{P}(a ; b)$ with a zero set $\Lambda$ and an indicator segment $[c ; d] \subset(a ; b)$ of length $2 \pi D_{B M}(\Lambda)$ is unique if and only if it is principle and weakly localizable.

Proof. Without loss of generality we assume that

$$
b=-a, \quad d=-c=-\pi D_{B M}(\Lambda) .
$$

According to the said in the Introduction for the case, the statement holds when the system $\operatorname{Exp}_{\Lambda}$ is complete or has a finite defect in the space $L^{2}\left(-\pi D_{B M}(\Lambda) ; \pi D_{B M}(\Lambda)\right)$. Indeed, this condition for the system $\operatorname{Exp}_{\Lambda}$ is equivalent to the fact that the submodule $\mathcal{J}$ is principle and is of the form 1.5 .

If the system $\operatorname{Exp}_{\Lambda}$ has an infinite defect in $L^{2}\left(-\pi D_{B M}(\Lambda) ; \pi D_{B M}(\Lambda)\right)$, then the part of the statement concerning necessity is implied by Theorem $A$ and the fact that a principle submodule is always stable.

To justify sufficiency we note that if $J$ is a weakly localizable principle submodule, then

$$
\operatorname{dim}\left(\mathcal{H}(\varphi) \ominus H_{p o l}\right) \leqslant 1
$$

see the proof of Theorem 1 and it remains to apply Theorem A.

The duality principle allows us to provide an equivalent formulation of Corollary 1 in terms of $D$-invariant subspaces.

Corollary 2. A D-invariant subspace $W$ with a given discrete spectrum $(-\mathrm{i} \Lambda)$ and a residual segment $[c ; d] \subset(a ; b)$ of length $2 \pi D_{B M}(\Lambda)$ is unique if and only if it is of form (1.6) and admits a weak spectral synthesis (1.2). 
It follows from Theorem 1 that a weak localizability of the principle submodule in the module $\mathcal{P}(a ; b)$ generated by the function $\varphi \in \mathcal{P}_{0}(a ; b)$ can be studied a possibility of approximating functions $\Phi \in J(\varphi)$ by countable sequences functions from the set $\operatorname{Pol}_{\varphi}$.

To formulate an appropriate criterion, we introduce the following notations: $u(z)$ is the maximal subharmonic minorant of the function $\left(h_{\varphi}(\arg z)|z|-\ln |\varphi(z)|\right)$, where $h_{\varphi}$ is the indicator function $\varphi$,

$$
H_{u}=\left\{\omega \in H(\mathbb{C}): \quad\|\omega(z)\|_{u}=\sup _{z \in \mathbb{C}}|\omega(z)| e^{-u(z)}<+\infty\right\} .
$$

Theorem 2. The principle submodule $J_{\varphi}$ generated by the function $\varphi \in \mathcal{P}_{0}(\mathbb{R})$ is weakly localizable if and only if each function $\omega \in H_{u}$ is approximated by the polynomials in the norm $\|\omega\|^{\prime}=\sup _{z \in \mathbb{C}}|\omega(z)| \exp (-u(z)-2 \ln (2+|z|))$.

Proof. It is clear we need to prove only necessity.

Let $\omega \in H_{u}$ and $\mu_{0}$ be some zero of this function, then $\frac{\omega}{z-\mu_{0}} \in \mathcal{H}(\varphi)$. By Corollary 11 and Theorem A, either $\mathcal{H}(\varphi)=H_{\text {pol }}$ or

$$
\operatorname{dim}\left(\mathcal{H}(\varphi) \ominus H_{\text {pol }}\right)=1 .
$$

In the first case for some sequence of polynomials $\left\{q_{j}\right\}$ the relation holds:

$$
\frac{\omega}{z-\mu_{0}}=\lim _{j \rightarrow \infty} q_{j}
$$

in the space $\mathcal{H}(\varphi)$. By Lemma 1,

$$
\left\|q_{j} \varphi-\frac{\omega}{z-\mu_{0}} \varphi\right\|_{0} \rightarrow 0,
$$

where $\|\cdot\|_{0}$ is determined by formula (2.1) with $c=c_{\varphi}, d=d_{\varphi}$. This implies easily the convergence of the polynomials $\left\{\left(z-\mu_{0}\right) q_{j}\right\}$ to a function $\omega$ in the norm $\|\cdot\|^{\prime}$.

If identity (3.1) holds, then

$$
\left(\alpha_{0} \frac{\omega}{z-\mu_{0}}+\alpha_{1} \frac{\omega}{z-\mu_{1}}\right) \in H_{p o l},
$$

for some $\alpha_{0}, \alpha_{1} \in \mathbb{C}$, where $\mu_{1} \neq \mu_{0}$ is one more zero of the function $\omega$. By Lemma 1, some sequence of polynomials $\left\{p_{j}\right\}$ converges to the function $\left(\left(\alpha_{0}+\alpha_{1}\right) z-\left(\alpha_{1} \mu_{0}+\alpha_{0} \mu_{1}\right)\right) \omega$ in the norm $\|\cdot\|^{\prime}$.

If $\alpha_{0}+\alpha_{1}=0$, then the statement holds. Otherwise, letting $\beta=\frac{\alpha_{1} \mu_{0}+\alpha_{0} \mu_{1}}{\alpha_{0}+\alpha_{1}}$ and taking into consideration the Phragmén-Lindelöf principle and the definition of the function $u$, we see that the sequence of the polynomials

$$
\tilde{p}_{j}(z)=\frac{p_{j}(z)-p_{j}(\beta)}{\left(\alpha_{0}+\alpha_{1}\right) z-\left(\alpha_{1} \mu_{0}+\alpha_{0} \mu_{1}\right)}, \quad j=1,2, \ldots,
$$

converges to the function $\omega$ in the norm $\|\cdot\|^{\prime}$.

\section{BIBLIOGRAPHY}

1. A. Aleman, B. Korenblum. Derivation-invariant subspaces of $C^{\infty} / /$ Comput. Meth. Funct. Theor. 8:2, 493-512 (2008).

2. N.F. Abuzyarova. Spectral synthesis in the Schwartz space of infinitely differentiable functions // Dokl. Akad. Nauk. 457:5, 510-513 (2014). [Dokl. Math. 90:1, 479-482 (2014).]

3. A. Aleman, A. Baranov, Yu. Belov. Subspaces of $C^{\infty}$ invariant under the differentiation// J. Funct. Anal. 268, 2421-2439 (2015).

4. N.F. Abuzyarova. Spectral synthesis for the differentiation operator in the Schwartz space // Matem. Zamet. 102:2, 163-177 (2017) [Math. Notes. 102:2, 137-148 (2017).] 
5. N.F. Abuzyarova. Principal submodules in the module of entire functions, which is dual to the Schwarz space, and weak spectral synthesis in the Schwartz space// J. Math. Sci. 241:6, 658-671 (2019).

6. N.F. Abuzyarova. Some properties of principal submodules in the module of entire functions of exponential type and polynomial growth on the real axis // Ufimskij Matem. Zhurn. 8:1, 3-14 (2016). [Ufa Math. J. 8:1, 1-12 (2016).]

7. A. Baranov, Yu. Belov. Synthesizable differentiation-invariant subspaces // Geom. Funct. Anal. 29:1, 44-71 (2019).

8. N.F. Abuzyarova. On 2-generateness of weakly localizable submodules in the module of entire functions of exponential type and polynomial growth on the real axis // Ufimskij Matem. Zhurn. 8:3, 8-21 (2016). [Ufa Math. J. 8:3, 8-21 (2016).]

9. J. Sebastian-e-Silva. "On some classes of locally convex spaces important in applications" // Matematika. Sbornik Perevodov. 1, 60-77 (1957). (in Russian).

10. L. Hörmander. The analysis of linear partial differential operators I: distribution theory and Fourier analysis. Springer, Berlin, (1980).

11. I.F. Krasičkov-Ternovskiı̌. Local description of closed ideals and submodules of analytic functions of one variable. I, II // Izvestia AN SSSR. Ser. Matem. 43:1, 44-66 (1979). [Math. USSR-Izvestiya. 14:1, 41-60 (1980).]

I.F. Krasičkov-Ternovskiĭ. Local description of closed ideals and submodules of analytic functions of one variable. II // Izvestia AN SSSR. Ser. Matem. 43:2, 309-341 (1979). [Math. USSR-Izvestiya. 14:2, 289-316 (1980).]

12. N.F. Abuzyarova. Closed submodules in the module of entire functions of exponential type and polynomial growth on the real axis // Ufimskij Matem. Zhurn. 6:4, 3-18 (2014). [Ufa Math. J. 6:4, 3-17 (2014).]

13. N.F. Abuzyarova. Principal submodules in the Schwartz module // Izv. Vyssh. Uchebn. Zaved. Mat. 5, 83-88 (2020). [Russian Math. 64:5, 74-78 (2020).]

14. L. de Branges. Hilbert spaces of entire functions. N.J.: Prentice-Hall inc. 1968.

15. Yu. Belov. Complementability of exponential systems // C.R. Math. Acad. Sci. Paris. 353, 215-218 (2015).

Natalia Fairbakhovna Abuzyarova,

Bashkir State University,

Zaki Validi str. 32,

450076, Ufa, Russia

E-mail: abnatf@gmail.com 